\title{
Parameter identification of the elastic modulus of ground rock based on blasting using the first order adjoint method
}

\author{
T. Ishimoto \& M. Kawahara \\ Department of Civil Engineering, Chuo University, Japan
}

\begin{abstract}
In the case of tunnel excavation, it is important to investigate ground properties before staring construction. However, it takes much money and time to investigate the ground properties. Therefore, a numerical technique to solve the problem is proposed in this paper. The method is parameter identification, which is inverse analysis based on an optimal control theory. The main purpose of this research is to present the parameter identification of elastic modulus at the Kasakura tunnel site located in Fukui prefecture in Japan. The blasting is made at the tunnel face and the vibration of the blasting is measured at the observation points. These observation velocities are used for the performance function.

Keywords: finite element method, parameter identification, first order adjoint method, weighted gradient method.
\end{abstract}

\section{Finite element equation}

Applying the finite element method to the basic equation, the finite element equation discretized by the linear tetrahedral element is obtained as follows.

In this paper, indecial notation and summation convention with repeated indies are used. The basic equation is expressed by the following three equations; the equilibrium of stress equation, the strain-displacement equation and the stressstrain equation.

$$
\begin{gathered}
\sigma_{i j, j}-\rho b_{i}+\rho \ddot{u}_{i}=0, \\
\varepsilon_{i j}=\frac{1}{2}\left(u_{i, j}+u_{j, i}\right),
\end{gathered}
$$


190 Underground Spaces I

$$
\sigma_{i j}=D_{i j k l} \varepsilon_{k l}
$$

where $\sigma_{i j}, b_{i}, u_{i}, \ddot{u}_{i}$ and $\varepsilon_{i j}$ are total stress, body force, displacement, acceleration, and strain, respectively. The elastic stress - strain relation is expressed by $D_{i j k l}$ and can be written as follows;

$$
D_{i j k l}=\lambda \delta_{i j} \delta_{k l}+\mu\left(\delta_{i k} \delta_{j l}+\delta_{i l} \delta_{j k}\right),
$$

where $\delta_{i j}$ is Kronecker's delta, in which Lame's constants $\lambda$ and $\mu$ are written as:

$$
\begin{aligned}
& \lambda=\frac{\nu E}{(1-2 \nu)(1+\nu)}, \\
& \mu=\frac{E}{2(1+\nu)},
\end{aligned}
$$

where $E$ and $\nu$ are the elastic modulus and the Poisson ratio, respectively. The basic equations are solved on the following boundary conditions.

$$
\begin{array}{ll}
u_{i}=\hat{u}_{i}^{0} & \text { on } t=t_{0}, \\
\dot{u}_{i}=\hat{u}_{i}^{0} & \text { on } t=t_{0},
\end{array}
$$

where $\hat{u}_{i}^{0}$ and $\dot{u}_{i}^{0}$ are the specified values given at the initial stage. The boundary $\Gamma$ is divided into $\Gamma_{1}$ and $\Gamma_{2}$. On $\Gamma_{1}$ boundary, displacement is specified and on $\Gamma_{2}$ boundary, surface force $t_{i}$ is given:

$$
\begin{aligned}
& u_{i}=\hat{u}_{i} \quad \text { on } \quad \Gamma_{1}, \\
& t_{i}=\sigma_{i j} n_{j}=\hat{t}_{i} \quad \text { on } \quad \Gamma_{2},
\end{aligned}
$$

where $\hat{u}_{i}$ and $\hat{t}_{i}$ are specified values on the boundary, $n_{j}$ is the external unit normal to the boundary.

\subsection{Finite element equation}

Applying the finite element method to the basic equation, the finite element equation discretized by the linear tetrahedral element is obtained as follows;

$$
M_{\alpha i \beta k} \ddot{u}_{\beta k}+C_{\alpha i \beta k} \dot{u}_{\beta k}+K_{\alpha i \beta k} u_{\beta k}=\hat{\Gamma}_{\alpha i},
$$

Each matrix can be written as follows;

$$
\begin{aligned}
M_{\alpha i \beta k} & =\rho \int_{V} \delta_{i k} N_{\alpha} N_{\beta} d V, \\
K_{\alpha i \beta k} & =\int_{V} N_{\alpha, j} D_{i j k l} N_{\beta, l} d V,
\end{aligned}
$$




$$
\Gamma_{\alpha i}=\int_{V}\left(N_{\alpha} \rho b_{i}\right) d V-\int_{\Gamma_{2}}\left(N_{\alpha} \hat{t}_{i}\right) d \Gamma,
$$

The effect of damping can be expressed as follows;

$$
C_{\alpha i \beta k}=\alpha_{0} M_{\alpha i \beta k}+\alpha_{1} K_{\alpha i \beta k},
$$

where $N_{\alpha}$ is the linear interpolation function of the finite element method. $M_{\alpha i \beta k}$ and $K_{\alpha i \beta k}$ are mass and elastic matrix, respectively.

\section{Newmark $\beta$ method}

In this paper, the Newmark $\beta$ method is applied to the discretization in time. In the Newmark $\beta$ method, displacement and velocity at $(n+1)$ time are expressed as follows; $(n+1)$ time are expressed as follows;

$$
\begin{aligned}
& u_{\beta k}^{(n+1)}=u_{\beta k}^{(n)}+\dot{u}_{\beta k}^{(n)} \Delta t+\frac{1}{2} \ddot{u}_{\beta k}^{(n)} \Delta t^{2}+\beta \Delta t^{2}\left(\ddot{u}_{\beta k}^{(n+1)}-\ddot{u}_{\beta k}^{(n)}\right), \\
& \dot{u}_{\beta k}^{(n+1)}=\dot{u}_{\beta k}^{(n)}+\ddot{u}_{\beta k}^{(n)} \Delta t+\gamma \Delta t\left(\ddot{u}_{\beta k}^{(n+1)}-\ddot{u}_{\beta k}^{(n)}\right),
\end{aligned}
$$

where $\beta$ and $\gamma$ are set as 0.25 and 0.50 , respectively. Eqs. (16) and (17) are substituted into eq.(14), the following equations can be derived as follows;

$$
G_{\alpha i \beta k} \ddot{u}_{\beta k}^{(n+1)}=\hat{\Gamma}_{\alpha i}-H_{\alpha i \beta k} \ddot{u}_{\beta k}^{(n)}-L_{\alpha i \beta k} \dot{u}_{\beta k}^{(n)}-K_{\alpha i \beta k} u_{\beta k}^{(n)},
$$

where $G_{\alpha i \beta k}, H_{\alpha i \beta k}$ and $L_{\alpha i \beta k}$ are written as:

$$
\begin{aligned}
G_{\alpha i \beta k} & =M_{\alpha i \beta k}+\gamma \Delta t C_{\alpha i \beta k}+\beta \Delta t^{2} K_{\alpha i \beta k} \\
H_{\alpha i \beta k} & =(1-\gamma) \Delta t C_{\alpha i \beta k}+\left(\frac{1}{2}-\beta\right) \Delta t^{2} K_{\alpha i \beta k}, \\
L_{\alpha i \beta k} & =C_{\alpha i \beta k}+\Delta t K_{\alpha i \beta k}
\end{aligned}
$$

Calculating acceleration $\ddot{u}_{\beta k}^{(n+1)}$ by eq.(18) and substituting it into eqs.(16) and (17), displacement $u_{\beta k}^{(n+1)}$ and velocity $\dot{u}_{\beta k}^{(n+1)}$ can be obtained.

\section{Performance function}

In this paper, the parameter identification is defined as finding optimal value so as to minimize the performance function. The performance function is defined as follows;

$$
J=\frac{1}{2} \int_{t}\left(\dot{u}_{\alpha i}-\dot{u}_{\alpha i}^{*}\right) Q_{\alpha i \beta k}\left(\dot{u}_{\beta k}-\dot{u}_{\beta k}^{*}\right) d t,
$$

where $\dot{u}_{\alpha i}$ and $\dot{u}_{\alpha i}^{*}$ are the computed and observed velocities and $Q_{\alpha i \beta k}$ is the weighting diagonal matrix. 


\section{First order adjoint equation}

In this paper, the extended performance function $J^{*}$ is expressed as follows;

$$
\begin{aligned}
J^{*} & =\frac{1}{2} \int_{t}\left(\dot{u}_{\alpha i}-\dot{u}_{\alpha i}^{*}\right) Q_{\alpha i \beta k}\left(\dot{u}_{\beta k}-\dot{u}_{\beta k}^{*}\right) d t \\
& +\int_{t} \lambda^{T}{ }_{\alpha i}\left(F_{\alpha i}-M_{\alpha i \beta k} \ddot{u}_{\beta k}-C_{\alpha i \beta k} \dot{u}_{\beta k}-K_{\alpha i \beta k} u_{\beta k}\right) d t
\end{aligned}
$$

where $\lambda_{\alpha i}$ is the Lagrange multiplier. Taking the first variation of the extended performance function, the gradient of the performance function, the adjoint equation and terminal condition can be calculated. The first variation of the extended performance function $\delta J^{*}$ is expressed as follows; The first variation of the extended performance function $\delta J^{*}$ is derived as;

$$
\begin{aligned}
\delta J^{*} & =\int_{t}\left(\dot{u}_{\alpha i}-\dot{u}_{\alpha i}^{*}\right) Q_{\alpha i \beta k} \delta \dot{u}_{\beta k} d t \\
& +\int_{t} \delta \lambda^{T}{ }_{\alpha i}\left(F_{\alpha i}-M_{\alpha i \beta k} \ddot{u}_{\beta k}-C_{\alpha i \beta k} \dot{u}_{\beta k}-K_{\alpha i \beta k} u_{\beta k}\right) d t \\
& +\int_{t} \lambda^{T}\left(\delta F_{\alpha i}-M_{\alpha i \beta k} \ddot{u}_{\beta k}-C_{\alpha i \beta k} \dot{u}_{\beta k}-K_{\alpha i \beta k} u_{\beta k}\right) d t,
\end{aligned}
$$

where $\delta J^{*}$ is transformed to obtain the gradient of performance function with respect the elastic modulus.

$$
\begin{aligned}
\delta J^{*} & =\int_{t}\left(\dot{u}_{i}-\dot{u}_{i}^{*}\right) Q_{\alpha i \beta k} \delta \dot{u}_{\beta k} d t \\
& +\int_{t} \delta \lambda^{T}{ }_{\alpha i}\left(F_{\alpha i}-M_{\alpha i \beta k} \ddot{u}_{\beta k}-C_{\alpha i \beta k} \dot{u}_{\beta k}-K_{\alpha i \beta k} u_{\beta k}\right) d t \\
& +\int_{t} \lambda^{T}\left(\delta F_{\alpha i}-M_{\alpha i \beta k} \delta \ddot{u}_{\beta k}-C_{\alpha i \beta k} \delta \dot{u}_{\beta k}-K_{\alpha i \beta k} \delta \dot{u}_{\beta k}\right. \\
& -\left(\alpha_{1} K^{*}{ }_{\alpha i \beta k} u_{\beta k}+K^{*}{ }_{\alpha i \beta k} \dot{u}_{\beta k}\right) \delta E d t .
\end{aligned}
$$

where $K^{*}=K_{\alpha i \beta k} / E$. Integrating by parts, $\delta J^{*}$ is transformed as follows;

$$
\begin{aligned}
\delta J^{*} & =\left(\dot{u}_{\alpha i}\left(t_{f}\right)-\dot{u}_{\alpha i}^{*}\left(t_{f}\right)\right) Q_{\alpha i \beta k}-\left(\dot{u}_{\alpha i}\left(t_{0}\right)-\dot{u}_{\alpha i}^{*}\left(t_{0}\right)\right) Q_{\alpha i \beta k} \\
& -\lambda_{\alpha i}^{T}(t f) M_{\alpha i \beta k} \delta \dot{u}_{\beta k}\left(t_{f}\right)+\lambda_{\alpha i}^{T}\left(t_{0}\right) M_{\alpha i \beta k} \delta \dot{u}_{\beta k}\left(t_{0}\right) \\
& +\dot{\lambda}_{\alpha i}^{T}\left(t_{f}\right) M_{\alpha i \beta k} \delta u_{\beta k}\left(t_{f}\right)+\dot{\lambda}_{\alpha i}^{T}\left(t_{0}\right) M_{\alpha i \beta k} \delta u_{\beta k}\left(t_{0}\right) \\
& +\lambda_{\alpha i}^{T}\left(t_{f}\right) C_{\alpha i \beta k} \delta u_{\beta k}\left(t_{f}\right)+\lambda_{\alpha i}^{T}\left(t_{0}\right) C_{\alpha i \beta k} \delta u_{\beta k}\left(t_{0}\right) \\
& -\int_{t} \ddot{\lambda}_{\alpha i}^{T} M_{\alpha i \beta k} \delta u_{\beta k} d t+\int_{t} \dot{\lambda}_{\alpha i}^{T} C_{\alpha i \beta k} \delta u_{\beta k} d t-\int_{t} \lambda_{\alpha i}^{T} K_{\alpha i \beta k} \delta u_{\beta k} d t \\
& +\int_{t} \delta \lambda^{T}{ }_{\alpha i}\left(F_{\alpha i}-M_{\alpha i \beta k} \ddot{u}_{\beta k}-C_{\alpha i \beta k} \dot{u}_{\beta k}-K_{\alpha i \beta k} u_{\beta k}\right) d t
\end{aligned}
$$




$$
\begin{aligned}
& -\int_{t}\left(\ddot{u}_{\alpha i}-\ddot{u}_{\alpha i}^{*}\right)^{T} Q_{\alpha i \beta j} \delta u_{\beta j} d t \\
& -\int_{t} \lambda_{\alpha i}^{T}\left(\alpha_{1} K^{*}{ }_{\alpha i \beta k} u_{\beta k}+K^{*}{ }_{\alpha i \beta k} \dot{u}_{\beta k}\right) \delta E d t,
\end{aligned}
$$

The first variation of the extended performance function $\delta J^{*}$ should equal to 0 . Then, the adjoint equation and the terminal condition are obtained.

$$
\begin{gathered}
M_{\alpha i \beta k} \ddot{\lambda}_{\beta k}-C_{\alpha i \beta k} \dot{\lambda}_{\beta k}+K_{\alpha i \beta k} \lambda_{\beta k}+\left(\ddot{u}_{\alpha i}-\ddot{u}_{\alpha i}^{*}\right) Q_{\alpha i \beta k}=0 . \\
\lambda_{\alpha i}\left(t_{f}\right)=0, \\
M_{\alpha i \beta k} \dot{\lambda}_{\beta k}\left(t_{f}\right)+\left(\dot{u}_{\alpha i}\left(t_{f}\right)-\dot{u}_{\alpha i}^{*}\left(t_{f}\right)\right) Q_{\alpha i \beta j}=0,
\end{gathered}
$$

where $\ddot{\lambda}_{\beta k}\left(t_{f}\right)$ is the terminal condition of acceleration at the terminal time $t f$ and $\ddot{\lambda}_{\beta k}\left(t_{f}\right)$ is solved for using $\dot{\lambda}_{\beta k}\left(t_{f}\right)$ and $\lambda_{\beta k}\left(t_{f}\right)$.

$$
\begin{aligned}
\ddot{\lambda}_{\beta k}\left(t_{f}\right) M_{\alpha i \beta k} & =-Q_{\alpha i \beta k}\left(\dot{u}_{\beta k}-\dot{u}_{\beta k}^{*}\right) \\
& +\dot{\lambda}_{\beta k}(t f) C_{\alpha i \beta k} \\
& -\lambda_{\beta k}(t f) K_{\alpha i \beta k}
\end{aligned}
$$

The gradient of the performance function is derived as follows;

$$
\operatorname{grad}\left(J^{*}\right)_{\beta k}=\lambda_{\alpha i}^{T}\left(\alpha_{1} K^{*}{ }_{\alpha i \beta k} \dot{u}_{\beta k}+K_{\alpha i \beta k}^{*} u_{\beta k}\right) .
$$

\section{Weighted gradient method}

The weighted gradient method is applied as the minimization technique. The modified performance function is expressed as follows;

$$
K=J^{*}+\frac{1}{2} \int_{t}\left(X_{\alpha}^{(n+1)}-X_{\alpha}^{(n)}\right) W_{\alpha \beta}\left(X_{\beta}^{(n+1)}-X_{\beta}^{(n)}\right) d t,
$$

where $X_{\beta}$ is to be identified. The optimal condition of the modified performance function is as follows;

$$
\delta K=0
$$

The parameters can be updated at each iteration.

$$
W_{\alpha \beta} X_{\beta}^{n+1}=W_{\alpha \beta} X_{\beta}^{n}-\operatorname{grad}\left(J^{*}\right)_{\beta} .
$$

Using eq.(34), the parameter is updated by the iterative calculations. 


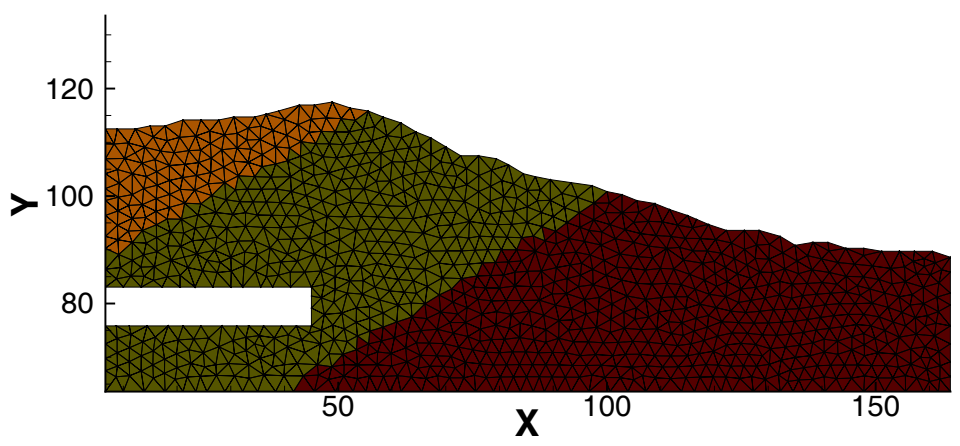

Figure 1: Finite element method.

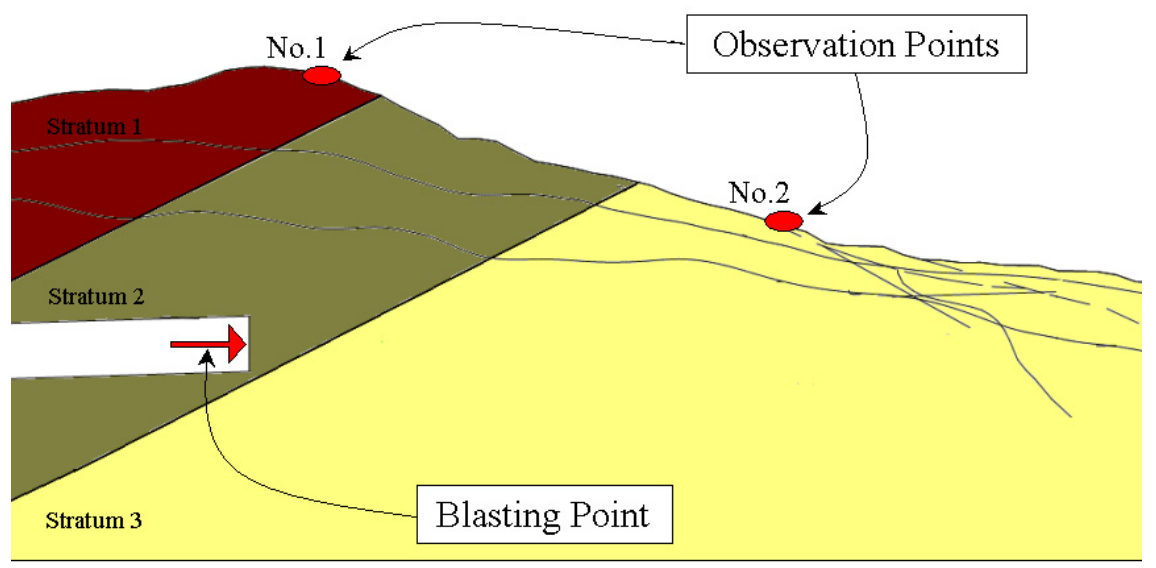

Figure 2: Computational domain.

\section{Numerical study}

The present method is applied to the Kasakura tunnel site. In the study, the actual observed data is employed. The purpose is to find parameter so as to minimize performance function.

The Kasakura tunnel construction site is located in Fukui prefecture in Japan. Fig. 1 illustrates the finite element mesh. Total number of nodes and elements are 1045 and 1934, respectively. There are three layers in this area. The elastic modulus is considered to be unknown in layer 2. Blasting force and observed points are shown in Fig.2. This blasting force is assumed as $1.0 \times 10^{8}\left[\mathrm{kN} / \mathrm{m}^{2}\right]$. The Poisson's ratio is 0.30 . The observed points is set at No.1 and No.2. The elastic modulus in the stratum colored in layer 2 is identified. 


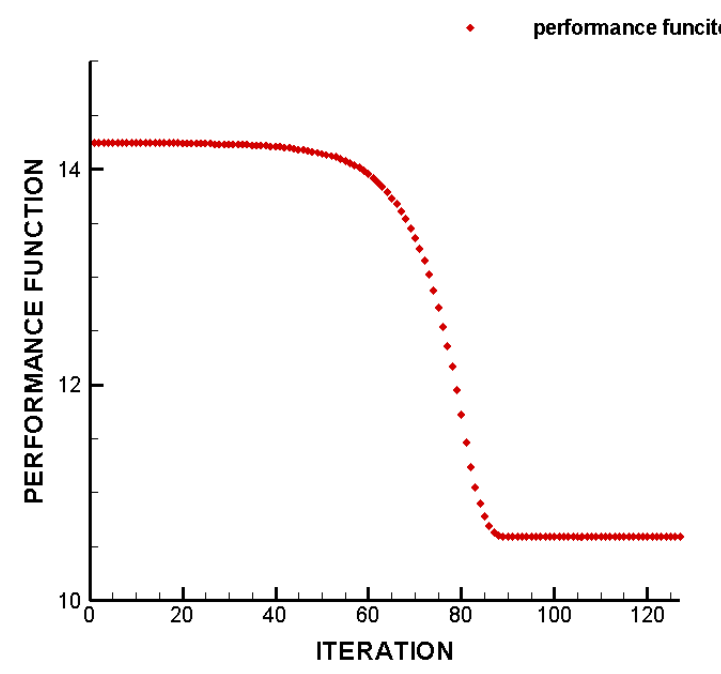

Figure 3: Performance function.

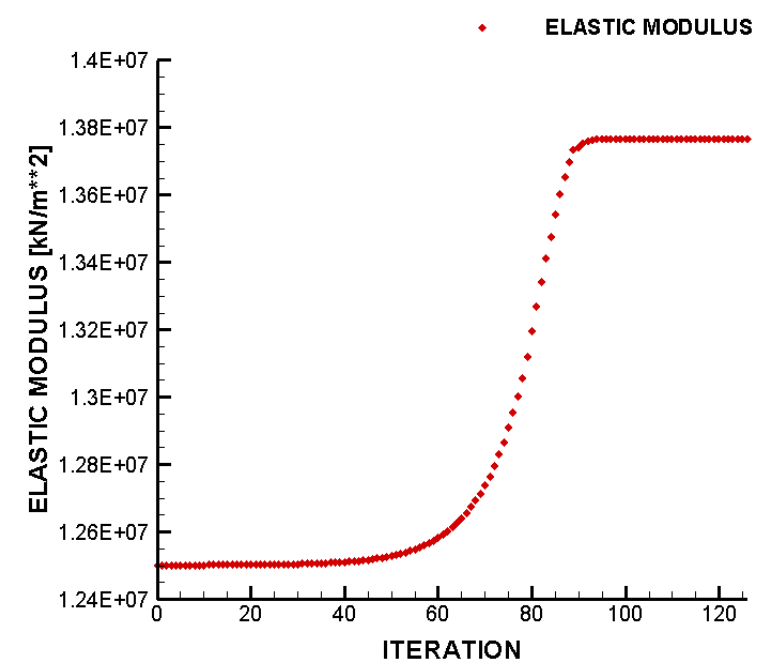

Figure 4: Elastic modulus.

\subsection{Basic equation}

The identification of the elastic modulus in the stratum colored in layer 2 is identified based on the velocities observed at observed points Nos. 1 and 2. Fig. 3 shows the variation of performance function. Fig. 4 shows the variation of the elastic mod- 
196 Underground Spaces I

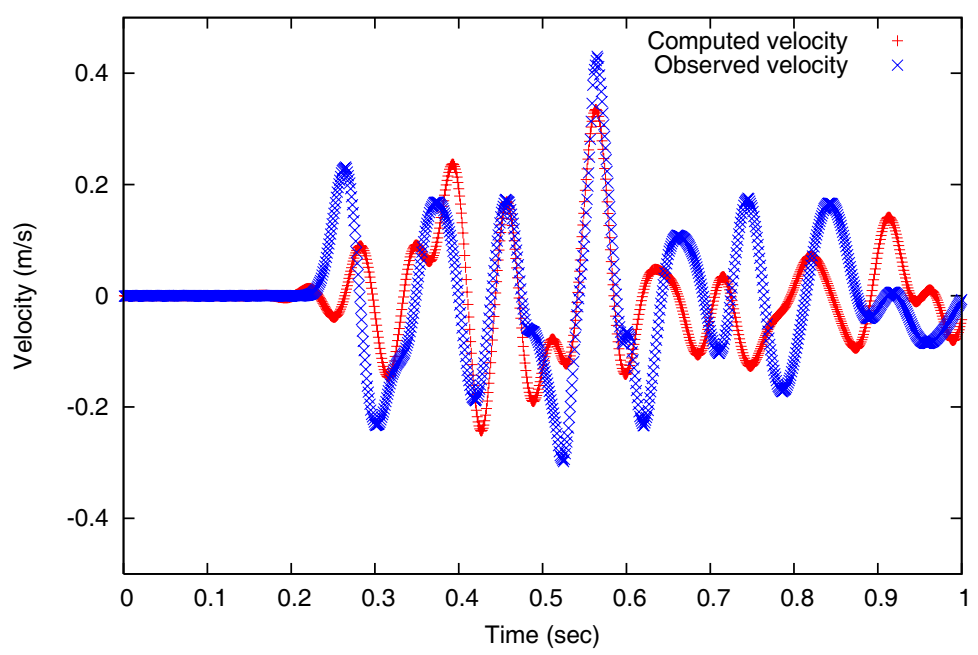

Figure 5: Y Velocity at point 1.

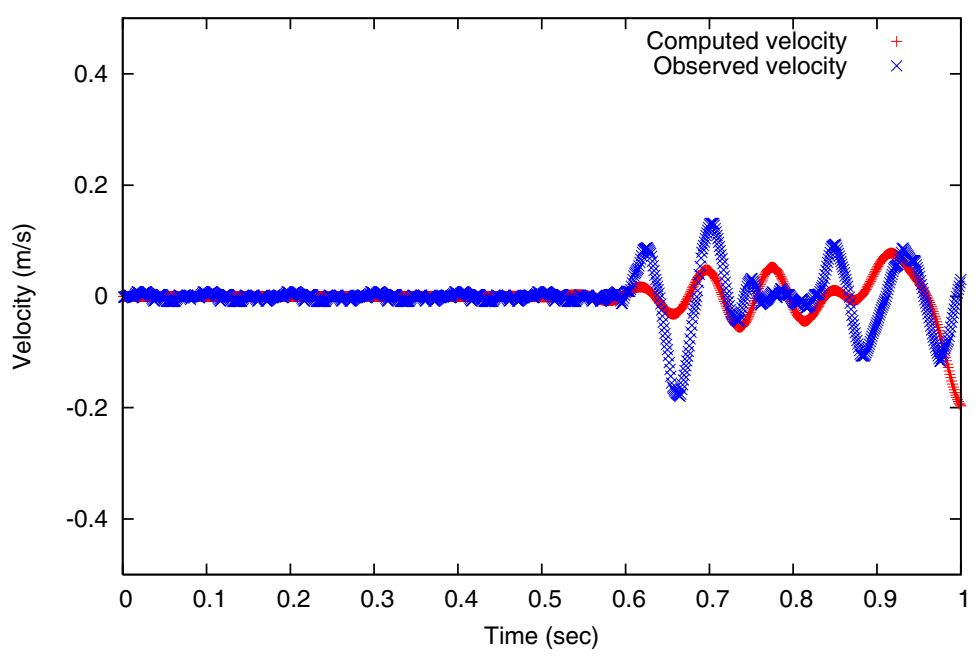

Figure 6: Y Velocity at point 2.

ulus. The final value of the elastic modulus computed based on the observed value is $1.38 \times 10^{8}\left[\mathrm{kN} / \mathrm{m}^{2}\right]$, which is in close agreement with the value obtained by the drilling tests. Figs. 5 and 6 show the variation of comparison velocity. Two data show almost same wave form. Few differences are influence of observed error and viscosity. 


\section{Conclusion}

The elastic modulus can be identified using the finite element method and the first order adjoint method of an optimal control theory. The performance function is defined as the square sum of the difference between computed and observed velocities. The elastic modulus is found so as to minimize the performance function. The elastic modulus is converged to the target value staring from the initial value. The performance function is converged to 0 . Thus, it is verified that the numerical method presented in this paper is correct and valid.

\section{References}

[1] I.M.Navon: Second-Order Information in Data Assimilation. American Meteorological Society, 629-648, (2002)

[2] R.H.F.Jackson and G.P.McCormick: Second-Order Sensitivity analysis in factorable programming.Theory and applications. Math. Program, 41, 1-28, (1988)

[3] R.H.F.Jackson and G.P.McCormick: The polyadic structure of factorable function tensors with application to high-order minimization techniques. Journal of Optimization Theory and Applications, 51, 63-94, (1986) 